\title{
MUJERES CASTELLANO-MANCHEGAS Y REPRODUCCIÓN ASISTIDA: UN ESTUDIO CUALITATIVO DESDE EL ÁMBITO SANITARIO
}

\author{
WOMEN INVOLVED IN ASSISTED REPRODUCTIVE TREATMENTS: A \\ QUALITATIVE STUDY IN THE MEDICAL CONTEXT
}

\author{
Beatriz Mañas Ramírez \\ Universidad Nacional de Educación a Distancia, Madrid. España/Spain \\ bmanas@poli.uned.es \\ Ana García Maestro \\ Goretti Calatayud Atencia \\ Servicio de Salud de Castilla-La Mancha (SESCAM), Albacete. España/Spain \\ agmaestre@sescam.jccm.es \\ gcalatayud@sescam.jccm.es
}

Recibido/Received: 27/03/2014

Modificado/Modified: 30/05/2014

Aceptado/Accepted: 27/06/2014

\section{RESUMEN}

Este trabajo surge de la necesidad de abordar los problemas de salud desde un enfoque holístico que permita comprender la perspectiva del propio sujeto en la definición, vivencia e interpretación de su situación y su contexto. Presentamos aquí el estudio cualitativo realizado sobre un colectivo de mujeres castellano-manchegas que demandan y reciben Técnicas de Reproducción Asistida (TRA). La práctica de grupos de discusión y su posterior análisis ha permitido captar las percepciones y representaciones de estas mujeres en torno a una serie de dimensiones que estructuran su propio autoconcepto, sus relaciones y su vivencia de un proceso que puede constituir la consecución o frustración de todo un proyecto vital. Tratándose de un ámbito donde se imbrican aspectos sociales, culturales, simbólicos y biomédicos, este enfoque de investigación pretende propiciar el trabajo conjunto de las ciencias sociales $\mathrm{y}$ de la salud para complementar y enriquecer ambos campos de conocimiento.

\section{PALABRAS CLAVE}

Investigación cualitativa, reproducción asistida, maternidad, fertilidad, sociología de la salud.

\section{SUMARIO}

1. El contexto de la investigación. 2. Métodos cualitativos en el análisis de la reproducción asistida. 3. El discurso de las mujeres. 3.1. Expectativa de la maternidad: "mujeres incompletas" en la "cuenta atrás". 3.2. Entorno, pareja y sexualidad. 3.3. Informaciones, nociones y prenociones. 3.4. La valoración del servicio sanitario: lo "público" y lo "privado". 4. Consideraciones finales. Bibliografía.

\section{ABSTRACT}

This research emerges from the necessity of facing health problems in a holistic way, able to capture the subjects' perspective about their situation and context, and the way they are perceived and understood. This paper presents the qualitative research we have developed about women living in Castilla-La Mancha who start or are already involved in Assisted Reproductive Treatments (ART). The 
implementation and analysis of discussion groups provide a rich understanding of these women's perceptions and representations about a series of dimensions that structure their own self-concept, their relations and their experience of a process which can provide or frustrate a whole vital project. In this context, where social, cultural, symbolic and biomedical aspects are involved, the cooperative work of social scientists and healthcare professionals can contribute to the advancement of both fields of knowledge, and to their mutual integration.

\section{KEYWORDS}

Qualitative research, assisted reproductive treatments, maternity, fertility, sociology of health.

\section{CONTENTS}

1. The research context. 2. Qualitative methods for the analysis of assisted reproduction. 3. Women's discourses. 3.1. The expectation of maternity: "incomplete women" in the "countdown". 3.2. Environment, partner and sexuality. 3.3. Information, notions and preconceptions. 3.4. The medical service assessment: the "public" and the "private". 4. Concluding remarks. References.

\section{EL CONTEXTO DE LA INVESTIGACIÓN}

El trabajo que aquí presentamos procede de la colaboración interdisciplinar de un equipo de profesionales de la sociología y de la enfermería para el análisis, desde un enfoque cualitativo, de un colectivo de usuarias del Sistema Público de Salud. En este sentido, el proceso de investigación plantea estímulos y retos importantes, tanto por la aproximación del personal sanitario hacia enfoques teóricos y metodológicos propios de las ciencias sociales y ajenos, en general, a su ámbito de actuación cotidiano, como por el interés y la pertinencia de la propia demanda que sustenta la investigación: la comprensión del proceso, la vivencia y las percepciones de las mujeres que solicitan Técnicas de Reproducción Asistida (TRA).

Esta colaboración entre especialistas de ámbitos sociales y sanitarios se fundamenta en el interés por abordar los problemas de salud desde una perspectiva integral, incorporando elementos analíticos que vayan más allá de los puros parámetros de diagnóstico biomédico. Ello implica utilizar herramientas que permitan un acercamiento holístico para comprender la forma en que el sujeto vive, define e interpreta su situación, procesos y síntomas. El uso de prácticas cualitativas de investigación social en el entorno sanitario puede enriquecer la comprensión de problemas médicos y de salud, que indudablemente presentan dimensiones sociales y simbólicas cuyo estudio requiere una aproximación pluridisciplinar. Especialmente relevante es el caso de las personas que demandan servicios de reproducción asistida, un colectivo cada vez más numeroso en los sistemas de salud público y privado, donde el proceso estrictamente médico se imbrica con toda una serie de expectativas y representaciones sobre la fertilidad, la maternidad, la familia, las relaciones de pareja o el proyecto vital, que modulan la propia vivencia del tratamiento e incluso pueden interferir en su seguimiento.

Los cambios socioeconómicos de las últimas décadas y sus consecuencias en las estrategias vitales y familiares han generado una demanda creciente de servicios médicos para el estudio y tratamiento de los problemas de fertilidad en la población. El estudio de la evolución de las tasas de infertilidad, [definida según la OMS como "la incapacidad de lograr un embarazo clínico después de 12 meses o más de relaciones sexuales no protegidas" (Zegers-Hochschild et al., 2010)] en distintos países durante el período 1990-2006 (Boivin et al., 2007) estimaba que más de 40 millones de mujeres de todo el mundo solicitan 
tratamiento. Estos datos agregados, que suponen una estimación conjunta para países de mayor y menor grado de desarrollo, cobran especial relevancia si atendemos a las consecuencias de los procesos económicos y sociales que han tenido lugar específicamente en los países industrializados, tales como la demora en la intención de tener hijos y el cambio en las estrategias de formación y composición familiar. El acceso a estudios superiores de capas de la población cada vez más amplias (muy particularmente en el caso de las mujeres), la inclusión de estas en el mercado laboral, y las incertidumbres provocadas por la precariedad laboral y el desempleo, especialmente en un contexto de crisis económica, retrasan la edad media de concepción favoreciendo la aparición de problemas reproductivos.

Tabla 1.Tasas de fecundidad en España según edad de la madre
Grupo $\mathbf{3 0 - 4 0}$ años
\begin{tabular}{c|rr} 
(Unidades: nacidos por $\mathbf{1 0 0 0}$ mujeres) \\
\hline Edad & $\mathbf{2 0 0 2}$ & $\mathbf{2 0 1 2}$ \\
\hline $\mathbf{3 0}$ años & $\mathbf{9 9 , 9}$ & $\mathbf{8 5 , 3}$ \\
$\mathbf{3 1}$ años & 102,9 & 95,3 \\
$\mathbf{3 2}$ años & 102,5 & 100,9 \\
$\mathbf{3 3}$ años & 94,5 & 100,1 \\
$\mathbf{3 4}$ años & 85,4 & 98,21 \\
$\mathbf{3 5}$ años & 73,0 & 92,2 \\
$\mathbf{3 6}$ años & 57,6 & 78,9 \\
$\mathbf{3 7}$ años & 44,6 & 65,7 \\
$\mathbf{3 8}$ años & 33,0 & 51,9 \\
39 años & $\mathbf{2 2 , 9}$ & $\mathbf{3 8 , 8}$ \\
$\mathbf{4 0}$ años & $\mathbf{1 5 , 6}$ & $\mathbf{2 8 , 8}$ \\
\hline
\end{tabular}
Fuente: Indicadores Demográficos Básicos (INE)

El análisis de la Encuesta de Fecundad Española de 1999 (Rodríguez y Ramírez, 2002) mostraba ya a comienzos del siglo XXI que la caída general de la fecundidad española afectaba muy particularmente a las mujeres menores de 30 años, quienes sin renunciar a tener hijos en su proyecto vital, aplazaban esa decisión en el tiempo. Esta tendencia general experimentada a lo largo de todo el siglo XX parece haberse profundizado durante la primera década del siglo XXI. Los indicadores del INE -Tabla 1- para el período 2002-2012 avalan la persistencia en el retraso de la procreación: mientras la tasa de fecundidad era de 15,6 nacimientos por cada mil mujeres de 40 años en 2002, la cifra asciende en 2012 a 28,8 nacimientos en esa misma edad.

Como puede observarse -Gráfico 1- si bien la tasa de fecundidad de las mujeres españolas de 30 años ha descendido un 15\% durante la última década, es significativo que durante ese mismo período casi se haya duplicado para las mujeres con edades entre los 39 y 40 años. Si consideramos el hecho de que la edad biológica de las mujeres va mermando significativamente su capacidad reproductiva a partir de los 35 años, la constatación de este retraso puede suponer un incremento de las personas con problemas reproductivos en las edades en que efectivamente se tiene intención de concebir. Los datos procedentes del V Congreso Internacional de Medicina Reproductiva (Requena, 2013) apuntan hacia ese sentido, situando actualmente la tasa de infertilidad en España entre el 15 y el 17 por ciento de la población. Si a ello añadimos el aumento de mujeres que, sin presentar a priori dichos problemas, se plantean la maternidad en solitario, es previsible que la demanda de tratamientos con TRA experimente un notable crecimiento en los próximos años. 
Diversos estudios han mostrado que las representaciones culturales sobre la fertilidad y la maternidad modulan las estrategias socioeconómicas y vitales. Así lo mostraba el estudio cualitativo de Bute y Jensen (2010) sobre las percepciones de las mujeres norteamericanas de bajos ingresos, quienes desarrollaban normas sociales propias respecto a la fertilidad y la sexualidad para asegurar el éxito económico y la subsistencia familiar. Pero también los procesos emocionales quedan afectados por aquellas representaciones cuando aparecen problemas reproductivos. C. B. Polis y L. S. Zabin (2012) señalan que las concepciones culturales y prenociones relacionadas con la infertilidad y la carga emocional asociada a este imaginario pueden interferir en la adaptación de la pareja a las TRA, condicionando incluso el éxito del tratamiento. En el mismo sentido, F. Omu (2010) ha destacado la importancia de la connotación social que cada cultura otorga al hecho de concebir, y a la responsabilidad que al respecto asigna a cada miembro de la pareja. En algunas sociedades el matrimonio no se considera consumado hasta que la pareja no trae un hijo al mundo, contemplando ese nacimiento como garantía de estabilidad. Cuando ello no ocurre, e independientemente de la causalidad real, se asume en muchos casos una "culpabilidad femenina" que arroja hacia la mujer una importante presión social y psicológica.

Gráfico 1. Incremento relativo de la tasa de fecundidad en España (año base: 2002)

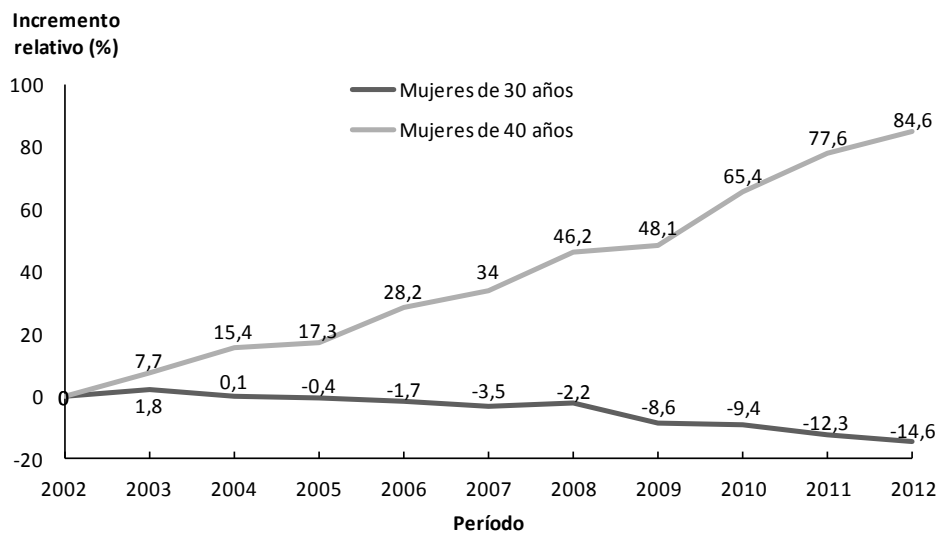

Fuente: Elaboración propia a partir de Indicadores Demográficos Básicos (INE)

La problemática de la infertilidad es un fenómeno complejo, atravesado por dimensiones sociales y culturales, cuyo abordaje, según Parada Muñoz (2006), debería asumirse de forma pluridisciplinar en la medida en que las probabilidades de éxito de las TRA no solo dependen de aspectos y procedimientos biomédicos. El acercamiento internacional desde enfoques integrales y cualitativos a los problemas de esterilidad/reproducción se ha desarrollado principalmente en estudios sobre jóvenes y minorías étnicas y culturales, fundamentalmente a partir de las líneas iniciadas en Estados Unidos y Canadá por Polis y Zabin, Bute y Jensen, y Wright. En España, la aproximación desde el entorno médico a la investigación social cualitativa aplicada a la concreta problemática de la reproducción asistida es limitada (cabe señalar los trabajos de López-Matheu, 2007). Sin embargo, hay que destacar la investigación sociológica y antropológica precedente en el ámbito más general de la salud, la enfermedad y la percepción del cuerpo -Menéndez (2005), Martínez (2004), Perdiguero y Cornellés (2000)y muy fundamentalmente los trabajos realizados por el grupo de investigación GIQS (Grupo 
de Investigación Cualitativa en Salud-UAM) integrado por Palmar, A., Pedraz, A., Ramasco M. y Zarco, J., sobre investigación cualitativa en salud (Palmar et al., 2013).

Desde estos planteamientos, y apostando por afianzar la colaboración pluridisciplinar en el análisis de problemas relacionados con la salud, la finalidad de la investigación que aquí presentamos es contribuir a la comprensión del proceso que atraviesan los personas que demandan técnicas de reproducción asistida, incorporando la perspectiva del propio sujeto inmerso en el tratamiento (sentido de la maternidad, percepción de su situación, conocimientos adquiridos sobre su problema, prácticas asociadas, reacciones afectivas, modulación de sus relaciones y de su perspectiva vital y familiar), complementando y enriqueciendo el conocimiento estrictamente biomédico.

\section{MÉTODOS CUALITATIVOS EN EL ANÁLISIS DE LA REPRODUCCIÓN ASISTIDA}

Numerosos estudios (Polis and Zabin, 2012; Bute and Jensen, 2010; Wright, 2004) han mostrado que la "infertilidad" es un tema socialmente delicado que se vive frecuentemente de manera vergonzante y del cual no es fácil obtener un discurso abierto y espontáneo. Acercarse a esta temática requiere una metodología de investigación que se adapte a la especial naturaleza de un tema sensible, cargado de connotación social, que pertenece mucho más al ámbito de las percepciones y del espacio simbólico que del conocimiento objetivo. Una temática, en definitiva, donde las dimensiones "social, simbólica, y emocional" se imbrican mutuamente y que aconseja ser abordada desde una perspectiva amplia y multidimensional.

La pertinencia del "enfoque cualitativo" para la investigación de este tipo de cuestiones se mostró en el ya clásico estudio sobre Las actitudes sociales ante el aborto realizado por el CIS (estudio 1394) mediante "grupos de discusión" durante los primeros años 80 (Ramos, 1981). A este estudio siguieron muchos otros que, tanto desde iniciativas públicas (CIS) como privadas, enriquecieron la investigación y el conocimiento sobre dimensiones socialmente sensibles, de difícil abordaje desde una perspectiva cuantitativa. Sin embargo, es poco frecuente en España que, desde un entorno propiamente médico, se utilicen técnicas procedentes de la antropología o la sociología para una comprensión más profunda de la situación que atraviesan los pacientes. Ello hace que el interés de nuestra investigación no solo proceda de la propia temática, sino de incorporar, desde una demanda generada en el ámbito sanitario, un enfoque cualitativo para el estudio de una problemática que además tiene indudable relevancia sociológica. Consideramos, por tanto, que el enfoque cualitativo es el más adecuado para abordar la especificidad de nuestro objeto de investigación, es decir, para comprender el discurso de las mujeres en proceso de reproducción asistida. Unas percepciones y vivencias difíciles de captar a través de la encuesta con cuestionario precodificado y el tratamiento estadístico de sus resultados, no solo por los problemas que tal método plantea para el tratamiento de temas delicados, sino por la dificultad de reflejar en el propio instrumento las dimensiones de análisis relevantes: estas solo pueden emerger en un contexto de investigación generativo que posibilite la creación discursiva, que permita, tal como señala J. Gutiérrez (2011), "dejar hablar” en lugar de "hacer hablar".

La elección de la metodología, que se concreta en la realización de grupos de discusión con pacientes y el posterior análisis cualitativo del discurso, ha supuesto retos importantes para el desarrollo de la investigación. En primer lugar porque se trata de un estudio que, aún siendo aprobado por los Comités de Investigación y Ético de la institución sanitaria pública 
de la cual surgía la demanda, hubo de realizarse sin financiación. Sin embargo, esta falta de recursos económicos se vio compensada con lo que finalmente constituyó el segundo reto de esta investigación: el trabajo de profesionales sanitarias con métodos y técnicas cualitativas, prácticamente desconocidas desde sus campos disciplinares. En este sentido, la predisposición hacia el conocimiento de nuevas metodologías y la implicación hacia el colectivo objeto de estudio con el que habitualmente el equipo de enfermeras matronas ha de trabajar, terminó estimulando sustantivamente la labor de la socióloga encargada de la investigación, enriqueciendo todo su desarrollo.

Las participantes de las reuniones fueron seleccionadas de entre el marco de pacientes de la institución sanitaria que facilitó el estudio, respetando lo máximo posible la condición de "homogeneidad intragrupal", es decir, seleccionando mujeres lo más similares posibles respecto a las variables que se consideraron potenciales generadoras de discursos diferenciados, y que definieron cada uno de los grupos.

\begin{tabular}{|l|} 
Cuadro 1. Variables para la formación de los grupos \\
\hline Variable 1: Fase del tratamiento \\
Criterio para la selección de participantes: \\
- Usuarias en Primera consulta \\
- Usuarias en Fases avanzadas del tratamiento \\
Variable 2: Tamaño del hábitat de residencia \\
Criterio para la selección de participantes: \\
- Usuarias residentes en hábitat urbano $(\geq 10.000$ hab.) \\
- Usuarias residentes en hábitat rural $(<10.000$ hab.) \\
Variable 3: Nacionalidad de origen \\
- Usuarias de origen español \\
- Usuarias de origen extranjero (Latinoamérica)
\end{tabular}

En efecto, se optó por realizar grupos con mujeres que se encontraban en primera consulta para evitar que la información y experiencia procedente del paso por el proceso pudieran contaminar sus percepciones previas. Igualmente, la experiencia de aquellas que ya han vivido el proceso de TRA debía analizarse con mujeres en fases avanzadas del tratamiento. La previsible riqueza discursiva de esta fase avanzada aconsejó la realización de dos grupos similares para asegurar la emergencia de todas las dimensiones discursivas relevantes. Por otra parte, las pautas socioculturales diferenciadas en entornos rurales y urbanos podían conducir a percepciones distintas sobre el concepto de fertilidad y maternidad, la relación con el entorno, y las expectativas respecto al proceso o al seguimiento de la terapia. De ahí que se decidiera realizar grupos específicos con mujeres residentes en núcleos pequeños de población o entornos rurales y municipios de mayor tamaño. Finalmente, dado que se constató que una proporción de las pacientes de las consultas de esterilidad tenía origen extranjero, consideramos que el estudio de las percepciones moduladas por posibles diferencias socioculturales de los respectivos países de origen podría enriquecer la investigación. Con el fin de asegurar un discurso fluido sin barreras para la comunicación se decidió formar uno de los grupos con mujeres extranjeras originalmente castellanoparlantes (finalmente latinoamericanas). De la combinación de estas tres variables, surge el diseño de los 5 grupos de discusión (GD) del estudio, celebrados en los meses de octubre-noviembre de 
2012 en una sala habilitada por un centro del servicio público de salud en Castilla-La Mancha:

\begin{tabular}{|c|c|c|c|}
\hline \multirow{2}{*}{} & \multicolumn{3}{c|}{ Tabla 2. Composición de los Grupos de Discusión } \\
\cline { 2 - 4 } & Nacionalidad & Tamaño del hábitat & Fase del tratamiento \\
\hline GD1 & Española & Mixto & Fase avanzada \\
\hline GD2 & Española & Mixto & Fase avanzada \\
\hline GD3 & Española & Urbano $(\geq 10.000$ hab. $)$ & Primera consulta \\
\hline GD4 & Española & Rural $(<10.000$ hab.) & Primera consulta \\
\hline GD5 & Extranjera (castellanoparlante) & Mixto & Mixto \\
\hline
\end{tabular}

Los grupos fueron moderados por la socióloga coordinadora del equipo, siempre acompañada por una investigadora enfermera matrona para propiciar su experiencia de campo con la dinámica grupal. Aunque se contó con un mínimo guión temático para la conducción de los grupos, se optó por un estilo de moderación abierto y flexible, permitiendo a las mujeres un contexto creativo de elaboración discursiva con el fin de generar categorías de análisis verdaderamente relevantes. La transcripción manual del registro también facilitó el primer contacto de las investigadoras con el discurso y su análisis, generando lo que $\mathrm{F}$. Conde denomina "conjeturas preanalíticas" (Conde, 2009), primeras ideas que van generando hilos y guías para el trabajo analítico posterior. La labor de análisis ha tenido como objetivo reconstruir los discursos de los colectivos de mujeres en los que se centra la investigación. Para gestionar la complejidad que representaba el material empírico obtenido, se dedicó una cuidadosa atención a la lectura y relectura de las transcripciones y a la selección de categorías de análisis que sintetizan los contenidos representativos del discurso a la vez que articulan toda su comprensión e interpretación. En este sentido, la riqueza discursiva y la relación de temáticas impiden que estas categorías puedan ser consideradas "compartimentos estancos", pues unas se relacionan con otras en la medida en que las percepciones, procesos y vivencias de estas mujeres se imbrican mutuamente conformando un tejido al que hay que dotar de sentido. Un sentido cuyo relato procedemos a exponer a continuación.

\section{EL DISCURSO DE LAS MUJERES}

La labor de análisis ha tenido como objetivo reconstruir los discursos de las mujeres en proceso de reproducción asistida. Ello implica un evidente esfuerzo de síntesis que pretendemos reflejar aquí ofreciendo las líneas discursivas fundamentales que articulan las percepciones y vivencias de estas mujeres (Los extractos de verbatim van acompañados, entre paréntesis, de la indicación del grupo de discusión al que pertenecen (G1, G2, G3, G4, G5), así como de la página de la transcripción en que se encuentran).

\subsection{Expectativa de la maternidad: mujeres "incompletas" en la "cuenta atrás"}

La idea de la maternidad se construye para estas pacientes como una "necesidad biológica" inherente al hecho de ser mujer, como una especie de llamada de la naturaleza, 
instintiva y ajena a cualquier control o voluntad. Una representación que las mujeres ilustran reiteradamente con una imagen: el deseo de "tocarse la tripa". Y si ello no se consuma, se vive de forma angustiosa como una tara, un defecto, un vacío que las transforma en seres incompletos y, por tanto, desesperanzados y preparados para una "muerte" simbólica. Es significativo que las alusiones a la maternidad siempre se ilustren discursivamente con el embarazo o, como mucho, con bebés recién nacidos, pero muy raramente con hijos adolescentes o mayores, a los que se menciona solo para explicitar los problemas o complicaciones que conlleva su educación, en un intento de compensar discursivamente la frustración por no vivir esa deseada primera experiencia: ser madre es "estar y sentirse embarazada", es "sentirse fértil" como "la tierra de la que emana vida"

Pero el ser una madre, yo creo que eso lo llevamos grabado en el genoma, ahí a sangre y a fuego $(\mathrm{G} 3,2)$.

Yo creo que como mujer o como madres que queremos ser, tenemos la necesidad de sentir la sensación de estar embarazadas y de ser madres $(\mathrm{G} 1,21)$.

Es que es una espiral de... porque si empiezas a pensar eso, luego piensas '¿y si no puedo?, y si no puedo, ¿qué hago?'. Y entonces tengo que... 'no voy a poder tener hijos, no voy a tener hijos, no vamos a tener'... Uffff, $Y$ entonces ya te ves vieja $y$ muerta y sola $(\mathrm{G} 3,24)$.

Yo me siento vacía. Si al levantarte de la cama no tienes nada que decir... voy a luchar por esto $(\mathrm{G} 1,45)$.

Pues el sentimiento, el sentirte... no sé, yo pienso que me gustaría... pues veo alguna embarazada y, lo que digo, me gustaría tocarme la tripa cuando las veo [...] $(\mathrm{G} 3,7)$.

El significante "adopción" aparece pocas veces. Aunque no dejan de considerarlo como posibilidad en última instancia, el deseo de vivir aquella primaria sensación impide que adoptar un niño sea una alternativa equiparable. La adopción se observa como una especie de maternidad "de consolación" cuya mera mención desde el entorno social de la mujer genera incluso reacciones de abierto rechazo o malestar:

[...] bueno, lo adoptas y luego lo voy a querer exactamente igual, pero ya no lo has sentido... no has tenido esa oportunidad de sentir, ya no con él, sino con ninguno, entonces... (G4, 15).

- Yo, lo que me hace mucho daño es cuando empiezan... sí que verdaderamente me hace daño, cuando empiezan: 'pues adopta' [expresado con ira]

-Ay sí, ese es el tema que más me hace daño

- Sí, sí

-A mí también

$-¿$ Y si no quiero adoptar? $(\mathrm{G} 1,21)$.

Solo una vez que se ha elaborado el discurso sobre la necesidad "biológica" de la maternidad emergen otras motivaciones, tales como evitar la monotonía que podría conducir a un estancamiento y cansancio en la relación de pareja o el deseo, particularmente en el caso de las mujeres rurales, de aumentar el número de miembros de la familia. Es en este colectivo y en el de mujeres latinoamericanas donde surge en mayor medida la preocupación por los cambios familiares de las últimas décadas, planteando con nostalgia conceptos familiares "de antaño", tales como la familia extensa, donde convivían un mayor número de miembros de distintas generaciones, y donde las abuelas, paradigmas del conocimiento doméstico ancestral, se implicaban en la crianza de los hijos. Las mujeres latinoamericanas perciben que 
las relaciones familiares son más frías y distantes en España que en sus países de origen, observando una pérdida de lo que consideran "valores esenciales" de respeto de los hijos hacia los padres. Es significativo que este colectivo observe con extrañeza la "normalidad" con la que en España se percibe el retraso en la edad de procreación de las mujeres, algo que en sus países de origen no está "bien visto":

[...] al venir aquí a España la... la idea es diferente, la idea es de que... se pospone la maternidad y... y no se ve mal el hecho de... luego de los 35 años empezar a tener hijos y... pues me atuve a eso... $(\mathrm{G} 5,2)$.

[...] estoy obsesionada... no con tenerlo ahora ya, ya, ya, sino... quedarme embarazada en algún momento. Y es también las familias en nuestros países, es que soy muy numerosas también, todo el mundo tiene hijos en cantidades... $(\mathrm{G} 5,4)$.

En general, a pesar de contemplar desde muy jóvenes la idea de la maternidad, las circunstancias sociales y personales vinculadas a las nuevas demandas y expectativas de las mujeres en el mundo académico, profesional, laboral y sentimental, han relegado la maternidad a un segundo plano durante un período importante de sus vidas. Esta circunstancia genera reacciones contradictorias: cuando aluden explícitamente a la "edad" como posible condicionante para la fertilidad, muchas de ellas se niegan a admitir que sea un factor determinante, encontrando siempre ejemplificaciones de familias de antaño en las que las mujeres tenían hijos a edades tardías. Sin embargo, cuando emerge el significante "tiempo" la elaboración discursiva deja claramente traslucir su obsesión por el "reloj biológico" que "juega en su contra" y que las sitúa en una continua "cuenta atrás" que va agotándose cada día. Esta doble dimensión implica, por un lado, la necesidad de trasladar fuera de ellas la "culpa" que sienten por hacer esperar la maternidad hasta no cumplir otras expectativas y, por otro, una gran ansiedad al considerarse efectivamente responsables de una serie de decisiones que obligan ahora a apresurar y a agilizar el proceso todo lo posible para que esa "cuenta atrás" no llegue a su fin. Esta desesperada necesidad de acortar el tiempo traslada continuamente el discurso hacia la "lentitud" con la que viven las listas de espera o el tiempo entre pruebas para realizar el tratamiento. Aquí se perciben diferencias entre la ilusión que expresan los grupos de fases iniciales del tratamiento, dispuestos a sacrificar lo que sea necesario de su vida cotidiana o de sus relaciones de pareja en la esperanza manifiesta de quedarse embarazadas, y la desesperación y frustración de las participantes de fases avanzadas, que han pasado por un largo periplo de pruebas y tratamientos, pero que fundamentalmente se enfrentan al pavor que les produce la posibilidad de confirmar del mayor de sus temores: la imposibilidad de procrear.

[...] yo siempre he tenido claro que quería tener hijos, y cuando conseguí tener una casa y casarme, y parece que la situación se estabilizó un poco, pues... nos planteamos ser padres enseguida porque siempre hemos querido tener hijos, entonces... te quedas si como 'i¿por qué yo no?! [enfatiza] $(\mathrm{G} 3,3)$.

El tiempo sobre todo, porque es que ya son... mucho tiempo, ya son casi dos años que estoy en este tratamiento y nada, ni una solución (G5, 31).

Un elemento fundamental del relato es la "presión" hacia la maternidad que manifiestan desde distintos niveles. Niveles que van desde las normas sociales y culturales a través de las que han interiorizado una determinada representación de la figura femenina, todavía anclada en su función como madre, pasando por el entorno más cercano, fundamentalmente explícito en el caso de las mujeres rurales, donde conocidos, familiares, amigos, etc. ejercen continuas injerencias proyectando hacia ellas sus expectativas sobre el proyecto de vida que estas 
habrían de llevar u opinando sobre el éxito o fracaso del tratamiento, hasta finalmente una presión personal, vivida muy internamente, descrita casi como una especie de "pulsión" que impone el deseo de ser madres a toda costa. Un deseo que juega en muchas ocasiones en contra de sus emociones, hasta el punto de describir su nula tolerancia a "ver bebés" a su alrededor, que les hablen de ellos, o incluso contemplar anuncios televisivos que los muestren.

[...] yo estaba harta de... ‘y cuándo vais a tener nenes?’, 'y vosotros ¿y por qué vosotros no vais a tener hijos?’ [...] y ya les digo: 'iporque no podemos!' $(\mathrm{G} 3,6)$.

Pero es que en mi pueblo hay mucha maldad: 'mira esa...' $(\mathrm{G} 4,42)$.

Es que, claro, es que en los pueblos está todo más controlado $(\mathrm{G} 4,43)$.

Ahora veo un niño y poco más que digo: '¡niño, quita, que no te quiero ni ver!', porque he reaccionado de manera rabiosa, de manera rabiosa $(\mathrm{G} 1,14)$.

El proceso emocional que atraviesan estas mujeres es muy complejo, pues todas las dimensiones de su vivencia afectan y se ven afectas por él. Fundamentalmente las que se encuentran en fases avanzadas describen un relato que puede compararse a un "duelo" con sus distintas fases, durante el cual experimentan numerosos cambios de ánimo, ansiedades y otras emociones hasta que finalmente parecen racionalizar la situación describiendo un estado más o menos adaptativo, en el cual, aunque el problema reproductivo siga ahí, son capaces de contemplar otras formas de disfrute relacionadas con vivencias compensatorias. Este proceso emocional es transversal, es decir, afecta a todas y cada una de las demás dimensiones de la vida de la mujer, y conscientes de ello, a menudo solicitan atención especializada por parte de profesionales que puedan acompañarles en el transcurrir del proceso médico.

Yo creo que en estos tratamientos haría falta... yo sé que el SESCAM está como está por los recortes y tal... una psicóloga. Un psicólogo, allí a tu lado (G1, 7).

Son significativas las reacciones que emergen en la dinámica: miedo, ansiedad, autoculpa, desesperanza, frustración, ira, rabia... emociones que, según las profesionales sanitarias, pueden incluso dificultar el éxito de las TRA. El discurso de los grupos de primera consulta y de fases avanzadas puso de manifiesto que, en relación a la esperanza que tienen depositada en el tratamiento y la aceptación de su posible infertilidad, se produce un cambio madurativo a lo largo del proceso. La clave para entender la relación de estas emociones con el posible y temido veredicto final sobre la imposibilidad de su maternidad, es que lo que se trunca no es solo tener un hijo, sino todo un proyecto de vida fraguado desde la infancia que forma parte de su autoconcepto como mujeres completas y que ahora se frustra. Es necesario entonces encontrar otros anclajes que, aunque aparecen discursivamente en un intento de racionalizar el escenario ("valorarse más a sí mismas", "reforzar el vínculo con la pareja", "vivir la vida", "dedicar más tiempo a ellas mismas") solo pueden entenderse como compensaciones de difícil adaptación a su actual situación.

Sí, sí, sí, sí, te haces muchas ilusiones y claro, pufff, para abajo... y caí, caí en picado. Y claro, ahora mismo estoy en plan... rabioso, como diciendo yo paso de todo' $(\mathrm{G} 1,29)$.

[...] por mucho que digas 'yo voy a vivir la vida', 'yo voy a pasar de todo', 'todo el tiempo es mio', 'todo el dinero que gano es para mi'... tal y cual... al final el problema lo tienes $(\mathrm{G} 1,14)$. 


\subsection{Entorno, pareja y sexualidad}

Las relaciones personales de las mujeres afectan y, a su vez, se ven afectadas por su vivencia del diagnóstico y por su proceso de tratamiento. A pesar de manifestar una genérica necesidad de apoyo, la gran mayoría prefieren no contar lo que les ocurre o llevar en secreto su tratamiento, evitando de esta manera los comentarios o consejos que terminan por incrementar la presión a la que acabamos de aludir. Sin embargo, se percibe una diferencia entre las usuarias urbanas de primera consulta, que manifiestan tener menos problemas para contar a su entorno cercano la situación que están atravesando, y las mujeres en fases avanzadas, que prefieren no hablar del tema debido al hartazgo y la decepción que les produce enfrentarse sin éxito a las distintas fases del tratamiento, algo que tendrían que reconocer públicamente como un fracaso. Como hemos señalado, en los pequeños núcleos de población el contacto con personas del entorno se vive con tintes paranoides, independientemente de la fase del tratamiento, pues aunque las relaciones no entrañen afinidades reales, el propio contexto hace inevitable la proximidad y la sensación de "control" ajeno. Esta necesidad general de aislamiento comunicativo tiene consecuencias en la inhibición de sus relaciones sociales, aunque ellas lo asumen y están dispuestas a aceptarlo como una especie de protección hacia el estigma y la vulnerabilidad con que se auto-perciben en comparación con otras mujeres.

Yo entiendo que... que el problema es mí... y que la gente no tiene por qué saber lo que a mí me pasa $(\mathrm{G} 2,47)$.

[...] es bueno que lo cuentes, sobre todo como hemos dicho, así, a amigas, gente muy... gente más cercana, que te pueda ayudar un poco... $(\mathrm{G} 3,6)$.

$[\ldots]$ yo tuve que dejar de ver a mis amigos $(\mathrm{G} 2,14)$.

No te apetece ver a nadie $(\mathrm{G} 2,14)$.

Muy relacionada con esta presión se encuentra la situación laboral de las mujeres que trabajan fuera del hogar. Aunque, en general, se asume que las empresas deben ofrecer posibilidades para conciliar el trabajo con el tratamiento (excedencias, reducciones de jornada, bajas, etc.) solo cuando las participantes admiten ser "sus propias jefas" asumen con tranquilidad que estas ventajas son posibles. En general, las mujeres perciben el paso por una TRA como amenazante para su puesto de trabajo dado que necesitan ausentarse para muchos de los procedimientos. Es clave aquí la diferencia entre la estabilidad de "las funcionarias" y la dificultad de aquellas que no gozan de un trabajo de duración indefinida, pues solo para las primeras la conciliación se percibe como una posibilidad real. A la hora de plantear a jefes o superiores la posibilidad de ausentarse para realizar el tratamiento, encontramos discursos diferenciales dependiendo del tipo de relación que mantienen, pero sí se considera de forma general que la empatía de estos es fundamental para facilitar o dificultar las condiciones reales de la mujer en el seguimiento de su proceso.

Además, encima claro, los turnos, que yo tengo que ir... mi madre que me lleva la medicación al trabajo, porque yo no la puedo tener alli 8 horas en una taquilla. Porque tiene que estar al frío (GD, 18).

Yo les digo ahora mismo 'voy a hacerme esto', y sé, sé que ellos pensarían en echarme $(\mathrm{G} 2,17)$.

El discurso de las mujeres en relación a la pareja surge fundamentalmente cuando es provocado por la moderadora de las reuniones, ya que el Sistema Sanitario Público que facilitó el estudio exige como criterio de admisión a los tratamientos que se trate de parejas heterosexuales, bien casadas o en situación de pareja de hecho. De ahí que todas las 
participantes tuvieran pareja masculina. Raramente aparece de forma espontánea en la dinámica de las reuniones y ello es indicativo de la gran importancia que tiene en su vivencia del proceso; la representación de la pareja en esta situación constituye un tema muy delicado que, en muchas ocasiones, revela inseguridades e incertidumbres que interfieren en otro aspecto más del proyecto vital: sus relaciones sentimentales. En este sentido, hay que decir que es prácticamente inexistente la emergencia de adjetivos posesivos en plural en referencia a la situación imaginaria de tener u hijo: no hay "nuestro", sólo "mi" o "mío". Ello es muy significativo de la percepción del proceso presente e idealmente futuro, en el caso de un posible embarazo, como una situación propia y casi exclusiva de la mujer, donde en general se asume para el hombre un papel secundario. La representación dominante con la que se identifican es la "maternidad", no la "paternidad", que tiene otras connotaciones. No niegan que la pareja pase por ansiedades o incertidumbres, pero los roles y actitudes asociados a su masculinidad implican vivir las cosas "de otra manera". Salvo intervenciones puntuales, las participantes se sienten apoyadas por sus parejas, entendiendo este apoyo como "sentirse" comprendidas, acompañadas, y como una forma de compartir sus mismos deseos e ilusiones o involucrarse en el tratamiento. De manera general las mujeres refieren que sus parejas no exteriorizan lo suficiente sus sentimientos y que son poco comunicativos con ellas. Consideran que esta falta de comunicación puede deberse, entre otras cosas, a que la esterilidad es un tema especialmente tabú para los hombres, que temen ser vistos a ojos de los demás como "culpables" de la situación -sean o no ellos los responsables "clínicos" de sus problemas de fertilidad- y, por tanto, menos viriles. Comparándose con ellos -algo que insisten en manifestar- perciben mayoritariamente que la mujer sufre más por el proceso que atraviesan, pues al dolor psíquico se suma un inevitable componente físico que solo ellas pueden experimentar en su paso por las pruebas y tratamientos. Aun así, las mujeres consideran que tienen más recursos y fortaleza que los hombres para sobrellevar y superar ese proceso.

-... Se lo guardan y no lo exteriorizan, yo creo que el problema es ese, porque si supieran... [asienten] hablar y comentarlo y decir 'tengo este problema', yo creo que es... se sentirían mejor y estarían mas... pero... vamos, yo lo digo bajo mi punto de vista... porque conozco a mi marido y sé como es y...

- Tienen más tabúes a lo mejor en ese sentido... (G3, 16).

Los hombres la verdad es que ellos son muy fuertes y muy 'esto', pero para eso nosotras ¿eh?, [risas] para aguantar... somos más valientes en ese aspecto... $(\mathrm{G} 5,4)$.

Es importante observar cómo la situación influye en la vida sexual de la pareja. Fundamentalmente en los grupos de fases avanzadas, aunque también sea perceptible en los de primera consulta, las participantes viven su sexualidad como si fuera una parte más de su tratamiento, como una "sexualidad pautada" por el profesional médico que las atiende, desprovista del deseo que, por el contrario, si concebían en las relaciones sexuales previas en las que muchas de las mujeres recocían haber utilizado métodos anticonceptivos. Desde el momento en el que surgen las dificultades explícitas para lograr un embarazo, y muy fundamentalmente, desde que comienzan a someterse a técnicas de reproducción asistida, el sexo pasa de representarse como una manera de compartir placer a un puro instrumento para conseguir el ansiado embarazo. Muchas de ellas reconocen que el sexo tiene lugar por "obligación", son los "deberes" que les ponen desde la consulta, y salvo comentarios puntuales, parece que el disfrute de sus relaciones íntimas ha quedado olvidado en fases anteriores de su relación de pareja. 
...Pero aunque tengas que poner las mejores de tus sonrisas y decir 'venga, va, que no tenga que llegar otra vez a los médicos, a ver si nosotros somos capaces' [...] y para mis adentros dije 'madre mía, si supieses qué calvario que llevo yo, todos los días sin querer' $(\mathrm{G} 1,37)$.

Cada dos días, cada tres más o menos, porque dices 'ea', si dicen que de seguido no, que por ejemplo, hoy, mañana, pasado que no, que no, que no, porque eso se tiene que regenerar... $(\mathrm{G} 3,29)$.

Que ya las tienes programadas [refiriéndose a las relaciones sexuales] o sea, ayer lo hice, mañana no, ¿cuándo tiene que ser? Pasado mañana, o sea, porque... tiene que ser programado $(\mathrm{G} 3,30)$.

Aún así, hay intervenciones que tratan de poner en valor la sexualidad como forma de compensar el hecho de no poder quedarse embarazadas, aludiendo a la "libertad" que supone no tener que preocuparse por usar métodos anticonceptivos o a la posibilidad de enriquecer la vida sexual con su pareja a través de nuevas prácticas, en un intento de "probar" si con ello aumentan las probabilidades de un posible embarazo

Pues mira yo le miro el lado bueno porque como no me puedo quedar embarazada lo puedo hacer las veces que quiera y cuando quiera (risas). Y ya está y ya a cada momento disfruto $(\mathrm{G} 4,51)$.

\subsection{Informaciones, nociones y prenociones}

Existe una necesidad imperiosa y generalizada de conocer el diagnóstico de su infertilidad. Uno de los elementos que origina mayor ansiedad a estas mujeres es ignorar el motivo por el que no pueden quedarse embarazadas. Si no saben cuál es la causa entienden que su problema está fuera de control y no puede encontrase una solución. Hasta tal punto llega esa inseguridad que muchas de ellas prefieren que se les descubra un problema de salud clínico antes de que las pruebas sigan mostrando que "todo está normal". Si la medicina no sabe encontrar "una patología", la esperanza se pierde.

Cuando me dijeron que podía ser por la sangre, en junio me lo dijeron que me iban a hacer la prueba, yo dije... mira, rezaba, digo 'ojalá sea por eso', y que me digan 'es por esto', y que me den una solución $(\mathrm{G} 1,32)$.

Debido a ello, tratan de conocer hasta el más mínimo detalle todo lo referente a su proceso de tratamiento, necesidad que se hizo evidente en la dinámica grupal cuando las participantes dedicaban buena parte de la reunión a describir pormenorizadamente su propio proceso, interpelándose frecuentemente entre ellas para comparar las respectivas situaciones. A través del discurso ajeno tratan de saber qué pueden esperar, comprobando si los servicios que están recibiendo son equivalentes a los del resto de mujeres, o que no hay equivocaciones en el abordaje médico de su problema. El relato del propio proceso es uno de los contextos discursivos donde en mayor medida se alude al significante "tiempo" comentado anteriormente, que ahora se convierte en un elemento fundamental de comparación entre las mujeres: "tiempo" como cuenta atrás, "tiempo" largo y tedioso con interminables listas de espera, "tiempo" entre los distintos ciclos, y "tiempo" cuya celeridad solicitan activamente para "llegar a tiempo" y concluir felizmente el tratamiento.

Yo pasé muchas decepciones. Y además yo tuve cuatro inseminaciones antes de la in vitro, y no os imagináis, bueno, a lo mejor lo habéis pasado también alguna de vosotras, o sea, cuatro inseminaciones, cuatro tratamientos, cuatro sí o no... (G2, 23). 
Que las listas de espera sean largas... que te mina mucho la moral, que te dicen 'venga en junio' entras, pues hasta dentro de un año o año y medio, y espérate que con los recortes a lo mejor se alarga mas la lista... (G3, 37).

Las participantes desarrollaron un discurso abierto sobre sus nociones acerca de lo que creen que influye o no en la fertilidad. Estas fases se acompañaban frecuentemente de risas y de un lenguaje en tono jocoso, fundamentalmente por la imbricación de elementos místicos y sexuales de la cultura popular en sus explicaciones. Una fase de las reuniones, sin duda, muy interesante, y muy difícil de explicitar de haber utilizado otras técnicas de interrogación. La mayoría de las mujeres conoce bien su ciclo menstrual, los cambios en su cuerpo tanto a nivel físico como psíquico, pero en la mayoría de los grupos -y aquí no hay diferencia, tal como se pensaba en un primer momento, entre las mujeres rurales y urbanas- se habla de productos diversos con supuestas cualidades propiciatorias de la fertilidad, tales como complejos vitamínicos, productos "naturales" o determinados alimentos. Entre estos productos nombran explícitamente "las carnes rojas", "el caldo de cáscara de castaña", "infusiones cíclicas", "el ginseng", etc., todos ellos conocidos fuera de la consulta médica, por canales informales o directamente a través de Internet. Aun siendo conscientes de que buena parte de ellos pueden tener una eficacia limitada, prueban absolutamente todo lo que "se dice" que funciona, venga de donde venga la información. Pero no se trata solo de productos adquiribles, también prueban todas aquellas prácticas sexuales que, "según se dice", pueden conducir con mayor facilidad a un embarazo.

... Indagando, indagando, encontré lo de las infusiones cíclicas que son unas infusiones, una mezcla de hierbas que te tienes que tomar en el momento que te baja el periodo hasta que vuelves a ovular, una serie de plantas todas mezcladas, hervidas y te las tomas todos los días un vaso. Y luego, antes de que te de la regla, pues otra... otra mezcla de infusiones. En Internet dice que va genial [...]. (G1, 41).

[...] Entonces dicen que para eso había unas posturas que era más fácil que te quedaras embarazada y tal, de cucharita, de no sé qué.... (G5, 25).

Los grupos de fase inicial coinciden con los grupos de tratamiento avanzado en señalar el estrés como factor más perjudicial para la fertilidad, en una clara proyección latente de su propio estado como causa generalizable. Aunque también aluden a la edad y al reloj biológico, es la ansiedad y la "obsesión" lo que para estas mujeres termina siendo el elemento fundamental de su problema, se haya detectado o no una causalidad médica. Otros factores aludidos, aunque en menor medida, son el sobrepeso, la alimentación, tabaco, alcohol y drogas, el café, los "disgustos", la contaminación, los productos químicos, el uso de móviles y la toma de anticonceptivos. Este último elemento es particularmente significativo pues, aunque se ha constatado que la toma de anticonceptivos no interfiere en la fertilidad una vez se abandona su consumo, estas mujeres proyectan hacia ellos una causa más de frustración, en la consciencia de que su uso en épocas anteriores se debe a una decisión personal de la que sí se sienten responsables.

Porque de hecho yo he estado con ansiedad hace años, me lo decían que eso... eso te bloquea parte del cuerpo. (G4, 31).

El tema de las comidas, el no fumar, el no beber, el no drogarse, todas esas cosas obviamente. (G5, 27).

O los anticonceptivos, porque te preguntan 'seguro que has estado un montón de años con los anticonceptivos'. (G4, 31). 
Una vez les han transferido los embriones, las mujeres de tratamiento avanzado suelen coincidir en los factores que consideran pueden provocar un aborto: no guardar reposo, ganar peso, realizar tareas domésticas como "usar la fregona", subir escaleras, o incluso hablan de la supuesta capacidad de la "Coca-cola" para disolver los embriones. En general, podemos decir que las participantes asumen los tópicos que circulan sobre las causas de infertilidad, pero no muestran seguridad al respecto. Ello es debido a que en muchos casos han tenido que pasar por un largo proceso en que se sentían "ignorantes" hasta llegar a un diagnóstico, y en otros casos, a que la causa sigue siendo desconocida, algo que se vive, tal como hemos comentado, con notable ansiedad.

Precisamente la demanda de información profesional relacionada con su problema constituye uno de los discursos absolutamente saturados y dominantes en todas las reuniones. Sin embargo, habida cuenta del detalle con el que muchas de ellas conocen su propio diagnóstico, su proceso y sus fases, esta demanda ha de entenderse como otra manifestación más de la frustración que les produce no conseguir un embarazo: "no entienden por qué". Y aunque la gran mayoría señalan que las consultas médicas deberían proporcionarles más información, finalmente el discurso desvela que lo que realmente solicitan no es tanto una mayor "cantidad informativa", sino que esta les sea transmitida "de otra manera", de forma más cálida, más empática, más humana, menos fría y con mayor consideración hacia el proceso psicológico que están atravesando.

[...] Creo que hace falta mucha información sobre... sobre el tema de los tratamientos y... y a dónde podemos consultar las dudas que tengamos en ciertos momentos. Porque a veces no sabes dónde tienes que acudir. Nos tendrían que dar más información. (G1, 2).

Fue horrible... la forma de decírtelo [...] O sea, la forma, el trato, para mí es tan importante como el propio tratamiento. $(\mathrm{G} 2,6)$

Sí, como que estás ahí preguntando y... iya está aquí la listilla preguntándome!, sí, sí, yo siento que eso...que como que les molesta un poco que uno les esté preguntando, insistiendo, que si no lo entiendes les vuelvas a preguntar. $(\mathrm{G} 5,14)$.

La necesidad informativa debe entenderse, por tanto, no como una carencia real de información, sino como una búsqueda incesante de elementos con los que poder identificarse para inferir cuál es su problema y qué pueden esperar a partir de ahora. De ahí que algunas de ellas, por temor a leer o escuchar algo que "no desean saber" prefieran no atender a ninguna otra fuente que no sea la del profesional médico. Por el contrario, la gran mayoría utilizan otros canales para buscar información, fundamentalmente Internet, que salvo excepciones, consideran una herramienta fácil y rápida para "enterarse" de lo que creen necesitar saber y para "compartir" en foros la propia situación. Al mismo tiempo, son conscientes de que gran parte de los datos de canales informales pueden resultar confusos e irreales, sobre todo si proceden del entorno social que les rodea. Es especialmente en los entornos rurales cuando la información recibida se considera falsa, basada en anécdotas y experiencias personales, incluso malintencionada, ya que sobre ella se proyecta el agobio que les produce la presión social del que estas mujeres buscan protegerse en muchos casos, sobre todo cuando se encuentran en fases avanzadas del tratamiento y su estado de ánimo es cada vez más vulnerable.

[...] Toda la información que yo recabé sobre todo este tema ha sido basada en Internet $(\mathrm{G} 1,2)$.

[...] Me fui a Internet, o sea me fui a Internet porque le pregunté a la médica de aquí y no me quedó claro $(\mathrm{G} 5,16)$. 


\subsection{La valoración del servicio sanitario: lo "público" y lo "privado"}

Aunque todas las participantes eran pacientes de la consulta de esterilidad del servicio público de salud, algunas de ellas habían iniciado igualmente tratamientos en clínicas privadas, particularmente en una institución de referencia de una ciudad cercana, y todas ellas tenían cierta percepción del sistema privado, hayan recurrido o no a él. En una primera elaboración discursiva se entiende que las "garantías" están en el sistema "de pago", denominación con la que popularmente se refieren a lo que no es un "sevicio público". El "pago", la contraprestación económica, implica una identificación de la paciente como "cliente", lo cual parece implicar una mayor confianza, no solo en el resultado final, sino en compensar todos los defectos y carencias que proyectan imaginariamente sobre la sanidad pública: pruebas específicas, mejores infraestructuras, mejor atención, mejor trato por parte del personal médico, menos listas de espera, descubrimiento e identificación "del problema" $\mathrm{y}$, en definitiva, mayor esperanza:

- Claro pero es que alli es otra cosa yo creo que lo privado es otra cosa, te tratan de otra manera, te explican de otra manera y todo.

- Porque lo pagas $(\mathrm{G} 4,35)$.

Sin embargo, las mujeres en fases avanzadas que han probado ambos sistemas refieren frecuentemente que las diferencias no son tan significativas, y que solo se centran en la calidad de las infraestructuras y los medios técnicos, fundamentalmente los laboratorios y las condiciones de las salas, que preservan en mucha mayor medida que en el sistema público, la intimidad de la pareja. Precisamente esta última cuestión suscita comentarios coincidentes de muchas de las participantes: las instalaciones del centro público se perciben poco adecuadas, poco íntimas, incluso indebidas frente al pudor que genera el desarrollo de las distintas pruebas. La concreción del discurso de las mujeres en este sentido es especialmente interesante por cuanto "revela" prácticas específicas asumidas por la institución sanitaria que están suponiendo problemas y situaciones incómodas tanto a las mujeres como a sus parejas. Las participantes comentan que la ubicación de la consulta de esterilidad les parece inadecuada por situarse justamente al lado de aquella donde se realizan las consultas de embarazo, y muy cerca de las consultas de pediatría, hecho que califican de tortura psicológica. También critican la sordidez y malas condiciones de los baños donde se recogen las muestras masculinas, solicitando a las propias moderadoras de la reunión trasladar esta queja a la institución pertinente, insistiendo en la habilitación de salas específicas para preservar la intimidad de la pareja.

Otra cosa que veo yo para machacarte más: ¿por qué tiene que estar esa consulta al lado de la consulta donde van todas las embarazadas a hacerse las ecografías? Que es que están ahí sentadas, las estás viendo y dices: 'bueno y ¿a mí cuándo me va a tocar estar en esta consulta? (G1, 29).

- [...] Yo te comento por él, dice 'claro me meten en ese cuarto así, chiquitísimo, con los carros pasando, el de mantenimiento que decía que si no subía, que si el ascensor... que si... 'y decía 'estos me abren a mi la puerta y me encuentran' (G4, 33).

En relación al trato del personal sanitario, que parece constituir otro de los grandes frentes abiertos en la percepción del servicio público, las mujeres de primera visita lo califican en general como muy bueno. Aunque sobre esta cuestión se generan discursos distintos tanto en los grupos de fases avanzadas de tratamiento como en el grupo de mujeres latinoamericanas, buena parte de las participantes denuncia la falta de sensibilidad y empatía de algunos profesionales sanitarios, explicitando en muchas ocasiones la metáfora "ganado" para referir 
la forma en la que en alguna ocasión se han sentido tratadas; una imagen coherente con el deseo, antes mencionado, de una relación más cercana y humana del personal médico a la hora de transmitirles la información sobre su diagnóstico y tratamiento. Igualmente, aunque son conscientes del recorte de recursos económicos que viene sufriendo la sanidad pública y que sus propios recursos no pueden costear en muchos casos un tratamiento privado, las mujeres solicitan imperiosamente (como desideratum dominante en todos los grupos) que se "acelere" el proceso, que se simplifiquen las listas de espera, que puedan ser atendidas y tratadas con mayor celeridad, que el proceso vivido en el sistema público no agote su inexorable "cuenta atrás".

[Hablando de su experiencia en el hospital público] Me han parecido personas súper agradables, súper cercanas que me han explicado todo muy bien, para mí la experiencia ha sido muy positiva $(\mathrm{G} 3,38)$.

[...] Esa es mi opinión, que te tratan como si fueras un número, ganado, y no te dan ninguna explicación $(\mathrm{G} 2,2)$.

[...] Y me urge el tiempo, no sé, por un lado eso, por otro lado el tiempo, que agilicen los tiempos... $(\mathrm{G} 5,30)$.

Lo que pasa que aquí van [chasquido]... Tienen muy poca... muy pocos medios, lo que es en el laboratorio, y hacen lo que pueden con lo que tienen $(\mathrm{G} 2,8)$.

\section{CONSIDERACIONES FINALES}

Hemos tratado de desarrollar aquí las principales conclusiones de la investigación que hemos llevado a cabo con mujeres en tratamiento de reproducción asistida. Ello constituye, a la vez, una limitación y una potencialidad: una limitación porque gracias a la riqueza del discurso hemos percibido que no se han agotado las posibles dimensiones a estudiar; una potencialidad porque esa misma riqueza sugiere la posibilidad de proseguir con esta línea de investigación, no solo explotando todavía más el corpus de verbatim recogido en los grupos realizados, sino también realizando nuevos grupos, afinando todavía más las variables para su formación. Hay que tener en cuenta que toda la investigación se ha realizado sin financiación económica, solo gracias a la motivación e implicación del equipo de investigación y al aval del centro sanitario que posibilitó el desarrollo del proyecto. En este sentido, una de las primeras conclusiones que podemos establecer es la pertinencia de realizar una investigación de mayor alcance que incorpore grupos de discusión masculinos, "los grandes olvidados" del proceso, que como nuestras participantes reconocían explícitamente, también atraviesan fases de cambio, tensión e incertidumbre.

Hay que destacar que lo que en principio se consideró como un elemento metodológico pertinente al objeto de estudio, terminó convirtiéndose en un cauce fundamental para la expresión de emociones y vivencias compartidas de las mujeres. Es decir, una decisión técnica (la elección de grupos de discusión) se reveló, sin pretenderlo a priori, como una situación terapéutica para las mujeres inmersas en un tratamiento de reproducción asistida. En la dinámica del grupo se producía un proceso de identificación mutua, de empatía, de "transferencia", en el cual hablaban abiertamente de temas o cuestiones que reconocían les costaba hacer visibles en otros contextos. En ese sentido, fue fundamental la elección de las variables para la composición de los grupos, pues la homogeneidad interna incidió todavía más en ese proceso de empatía e identificación. De hecho, fueron muchas las mujeres que manifestaron, al término de las reuniones, lo agradable que había sido la experiencia, lo "bien 
que lo habían pasado" o la necesidad de que se convocaran más reuniones como estas a las que pudieran asistir con regularidad.

El trabajo de los profesionales de enfermería que trabajan en las consultas de fertilidad se ve inevitablemente afectado por la creciente demanda de servicios de reproducción asistida, teniendo que adaptar sus planes de atención y cuidados. Además del interés de la problemática de la infertilidad desde sus dimensiones sociales y culturales, esperamos haber contribuido con este estudio a poner en valor la colaboración y cooperación de profesionales del ámbito médico y de las ciencias sociales, cuyo trabajo conjunto puede proporcionar, sobre este y otros temas relacionados con la salud, conocimientos y experiencias de gran interés para sus respectivos campos disciplinares.

\section{BIBLIOGRAFÍA}

BOIVIN, J.; BUNTING, L.; COLLINS, J. y NYGREN, K. (2007). "International estimates of infertility prevalence and treatment-seeking: potential need and demand for infertility medical care", en Human reproduction, 22 (6): 1506.

BUNTING, L. y BOIVIN, J. (2008). Knowledge about infertility risk factors, fertility myths and illusory benefits of healthy habits in young people, en Human Reproduction. 23 (8): 1858-1864.

BUTE, J. y JENSEN, R. (2010). "Low-income women describe fertility-related expectations: descriptive norms, injunctive norms, and behavior", en Health communication, 25: 681-691.

CIS - Investigaciones cualitativas, accesible en http://www.cis.es/cis/opencm/ES/1 encuestas/estudios /busqueda.jsp.

CONDE, F. (2009). Análisis sociológico del sistema de discursos. Madrid: Cuadernos Metodológicos (43) CIS.

GUTIÉRREZ BRITO, J. (2011). "Grupo de discusión: ¿prolongación, variación o ruptura con el focus group?", en Cinta de Moebío: Revista de Epistemología de Ciencias Sociales, 41: 105-122.

LÓPEZ-MATHEU, C., et al. (2007). "Los jóvenes y la reproducción asistida", en Cultura de los cuidados. 22: 70-79.

MARTÍNEZ, A. (2004). "La construcción social del cuerpo en las sociedades contemporáneas", en Papers, 73: 127-152.

MENÉNDEZ, E. (2005). "Intencionalidad, experiencia y función: la articulación de los saberes médicos", en Revista de Antropología Social, 14: 33-69.

OMU, F. E. y OMU, A. E. (2010). "Emotional reaction to diagnosis of infertility in Kuwait and successful clients' perceptions of nurses' role during treatment", en Nursing, 9: 1-10.

PALMAR, A. et al. (2013). La investigación cualitativa en salud. Retos y compromisos. Comunicación presentada en el XI Congreso Español de Sociología (FES). Madrid, 10-12 julio.

PARADA MUÑOZ, L. M. (2006). "Infertilidad y pareja: construcciones narrativas como horizonte para la intervención", en Revista Diversitas. Perspectivas en Psicología, 2 (1): 149-158.

PERDIGUERO, E. y CORMELLES, J. M. (eds.) (2000). Medicina y cultura: estudios entre la antropología y la medicina. Barcelona: Bellaterra.

POLIS, C. B. y ZABIN, L. S. (2012). "Missed Conceptions or Misconceptions: Perceived Infertility Among Unmarried Young Adults In the United States", en Perspectives on Sexual and Reproductive Health, 44 (1): 30-38.

RAMOS, R. (1981). "Informe-resumen de los resultados de una investigación sociológica sobre el aborto mediante discusiones de grupo", en REIS, 21: 243-254.

REQUENA, A. (2013). "La tasa de infertilidad llega al 17\% de la población y seguirá creciendo", en $L a$ Vanguardia (05/04/2013), accesible en http://www.lavanguardia.com/salud/20130405/54370951544/ta sa-infertilidad-llega-17-poblacion-y-seguira-creciendo.html.

RODRÍGUEZ SUMAZA, C. y RAMÍREZ ESTÉVEZ, G. (2002). “Actitudes y comportamientos de las mujeres jóvenes en materia de fecundidad", en Estudios de Juventud, 58: 1-11. 
WRIGHT, K. L. (2004). 'Definir l'infertililté: que signifie le terme 'infertilité' pour les cliniciens et pour leurs patients?", en Family Health International. Network en français, 23 (2): 4-8.

ZEGERS-HOCHSCHILD, F. et al. (2010). Glosario de terminología en Técnicas de Reproducción Asistida (TRA). International Committee for Monitoring Assisted Reproductive Technology (ICMART) y Organización Mundial de la Salud (OMS).

\section{Breve currículo:}

\section{Beatriz Mañas Ramírez}

Licenciada y Doctora en Ciencias Políticas y Sociología por la UNED. Profesora Ayudante Doctora en el Departamento de Sociología I de la UNED. Desarrolla su labor docente en el área de Métodos y Técnicas de Investigación Social y Estadística aplicada a las Ciencias Sociales. Ha realizado estancias de investigación en instituciones internacionales -EHESS (París), Universidad de UCLA (Los Ángeles), LSE (Londres), MSH (Nantes)- centrando sus intereses en la investigación cualitativa en el ámbito sanitario, y en los métodos para el estudio de la opinión pública, dando lugar a numerosas publicaciones $\mathrm{y}$ trabajos en Congresos nacionales e internacionales.

\section{Ana García Maestro}

Diplomada Universitaria en Enfermería. Especialista en enfermería obstétrico-ginecológica. Combina su labor profesional en el Servicio de Salud pública de Castilla-La Mancha (SESCAM) con sus intereses de investigación relativos al ámbito de salud integral de pacientes en tratamiento con técnicas de reproducción asistida (TRA). Ha participado en numerosos Congresos y Jornadas nacionales relacionados con las especialidades neonatal y obstétrico-ginecológica. Ha sido miembro del equipo investigador en estudios cualitativos relacionados con el estudio integral de la salud desde el ámbito sanitario.

\section{Goretti Calatayud Atencia}

Diplomada universitaria en Enfermería. Especialista en enfermería obstétrico-ginecológica. Desarrolla su actividad profesional en el Servicio de Salud de Castilla-La Mancha, concretamente en la sección maternoinfantil. Sus intereses de investigación se centran en la integración multidisciplinar para el estudio del proceso y seguimiento de pacientes, fundamentalmente en el área obstétrico-ginecológica. Ha participado en Jornadas relacionadas con su actividad profesional. Ha colaborado como investigadora en proyectos de investigación social cualitativa para el estudio de mujeres en tratamiento de reproducción asistida.

* Este trabajo solo puede entenderse desde el esfuerzo y la implicación colectiva de las integrantes del equipo de investigación: por orden alfabético, Goretti Calatayud Atencia, Ana García Maestro, Catalina Lillo Serrano, $M^{\mathrm{a}}$ Luisa Rodrigo Pérez y Nieves Sánchez Sánchez, enfermeras matronas del SESCAM (Servicio de Salud de Castilla-La Mancha), cuyo trabajo ha sido dirigido y coordinado por la doctora en Sociología Beatriz Mañas Ramírez. 